\title{
Embedded hardware for closing the gap between research and industry in the assistive powered wheelchair market
}

\author{
V. Canoz, M. Gillham, P. Oprea, P. Chaumont, A. Bodin, P. Laux, M.Lebigre, G. Howells, K. \\ Sirlantzis
}

\begin{abstract}
Literature is abound with smart wheelchair platforms of various developments, yet to date there has been little technology find its way to the market place. Many trials and much research has taken place over the last few decades however the end user has benefited precious little. There exists two fundamental difficulties when developing a smart powered wheelchair assistive system, the first is need for the system to be fully compatible with all of the manufacturers, and the second is to produce a technology and business model which is marketable and therefore desirable to the manufacturers. However this requires the researchers to have access to hardware which can be used to develop practical systems which integrate and communicate seamlessly with current manufacturer's wheelchair systems. We present our powered wheelchair system which integrates with $95 \%$ of the powered wheelchair controller market; our system allows researchers to access the low level embedded system with more powerful computational devices running sophisticated software enabling rapid development of algorithms and techniques. When they have been evaluated they can be easily ported to the embedded processor for real-time evaluation and clinical trial.
\end{abstract}

\section{INTRODUCTION}

There has been significant research undertaken in the field of smart powered wheelchair platforms over the last 30 years $[1,2]$. Whilst some platforms have continued to be used for research most have not, research between 2005 and 2013 numbers over 4,018 published papers [3]. There is a clear gap in the research-to-market chain of development, almost no publications on the subject, according to Garcia [3]. Previous work [3, 4] undertaken to bridge this gap used existing development hardware and sought to develop a proof-ofconcept system; however there were significant issues relating to full system integration due to unknown delays and input signal response timing in the existing powered wheelchair system, sensor and node latency, ranging data rates, cost and size [4].

Technology is becoming increasingly import in modern society, people are able to communicate with each other in many ways and information has become a highly desired commodity. Devices with different architectures and systems are able to interconnect using industry standard protocols, software, and hardware combinations such as Universal Serial Bus (USB), Ethernet, Wi-Fi, Bluetooth, and the Global

V. Canoz, P. Chaumont, A. Bodin, P. Laux, M.Lebigre are with the École nationale supérieure d'ingénieurs de Limoges, 16 Rue Atlantis 87068 Limoges, Cedex, France.

M. Gillham (M.Gillham@kent.ac.uk), P. Oprea, G. Howells, K. Sirlantzis (K.Sirlantzis@kent.ac.uk), are with the School of Engineering and Digital Arts at The University of Kent, Jennison Building, Canterbury, Kent, United Kingdom, CT2 7NT. Telephone +44 (0)1227 823246.
System for Mobile Communications (GSM). Whilst it may be fully acceptable to have delays of up to one second in the updating of position on the Satnav, the same is not true if the vehicle brakes take seconds to begin to work. This equally applies to the powered wheelchair, it is vital that any collision avoidance must be running in real-time, due to the close proximity to obstacles, and be able to pass that information to the wheelchair system so that the assistance is immediate; equally the wheelchair's location and battery state may need to be passed to the carer, on their mobile phone, so that they can monitor the person in their care.

The current (2011) United Kingdom (UK) census data quotes the population as 56,075,912. A survey of disability in Great Britain, carried out by the Office of National Statistics in 1989 [5], indicated that out of the UK population some $13 \%$ of the population had a disability of some kind, and that, of these, $69 \%$ had a 'locomotion problem' these percentages relate to the current number of UK disabled as 7,570,248 with 5,223,471 needing assistance with mobility. The survey estimated that $10 \%$ with a 'locomotion problem' used a wheelchair, and that $10 \%$ of those wheelchairs were powered. This would indicate there are around 52,000 powered wheelchair users in the UK, if they renew their wheelchair every ten years then there is a market of 5,200 units per annum. Extrapolating these figures across the European Union (with an estimated population of 510 million) would mean there are potentially around 51,000 units per annum, and across the United States of America (USA) with an estimated population of 320 million, some 32,000 units per annum.

The number of potential people who would benefit from a 'smart wheelchair' in the USA are between 13.5 and 15.4 million according to research by Simpson [6] he goes on to list various disabilities and injuries which affect the powered wheelchair user making their needs quite complex; Simpson lists 13 different diagnosed conditions, each with a specific range of some 14 symptoms, he also goes on to argue there are considerable difficulties in determining the exact needs and requirements for powered wheelchair users, collision avoidance, navigation assistance, and human input interface being examples of the typical areas of assistance required. Simpson concludes that "Investment in smart wheelchair research, therefore, has much greater potential impact than previously thought" he concludes, that there appears to be a very large un-met and unqualified need, and more research is required in order to assist industry to provide suitable and affordable technology.[6].

Garcia et al. [3] reports that industry have shown little interest because the returns do not justify the investment, he describes this as the inverted pyramid. This is where users 
who require the most assistance, and hence complex hardware, number the least and the highest number of users have the least assistance requirement; thus the market returns diminish as the assistive devices become more complex. They go on to say that there are only two systems currently available on the market, and only one of them is available to end-users. This is a line-following platform which the powered wheelchair can sit-on. In conclusion Garcia et al. state that for research to reach the market, hence end-user, a standardization needs to be undertaken; they suggest that a low-cost, system-integrated, modular interface device is needed to bridge the gap.

According to Simpson's research there appears to be far fewer people using powered wheelchairs than could benefit from having one, this implies that there may be a large market of potential powered wheelchair users who would be able/allowed to drive if they were given minimal assistance [7], this would invert the pyramid described by Garcia et al. and make assistive devices attractive to industry. Argall argues that this could be best demonstrated by developing low-cost modular hardware and software which can be added to commercially available wheelchairs [8] other research talks about the need to customize assistance for different disabilities, but they note the clinical necessity to keep the user in control $[9,10]$, this would require a human centered control methodology [11] which would mean that any system must operate in real-time to prevent causing any hindrance and distress to the users of the system.

In order to develop technologies, software, and algorithms to assist disabled powered wheelchair users, and to get that assistance to the end-user, requires a hardware interface which fully and seamlessly integrates into existing platforms as if it were the manufacturer's plug-and-play accessory. This device should also be capable of interfacing with other devices such as mobile phones and tablets, and finally should use low-cost components and sensors, and be robust and safe.

When industry develops new hardware accessories for existing systems it is quite common for the manufacturer of the proprietary system to allow third parties to access their system via a low-cost interface, which they provide, so as to minimize the development complexity and more importantly to maintain their intellectual property; our system connects to the two main manufacturers of powered wheelchair control system in just such a manner.

The current method manufacturers use to provide a safe and robust control system for the commercially available powered wheelchair is to use an open-loop approach, this relies upon taking the user joystick input and mapping that input to the motor input in such a way that the dynamic variables are constrained and the kinematic constraints are obeyed. These parameters are usually as follows, but by no means limited to:

- forward and reverse velocity

- forward and reverse positive acceleration

- forward and reverse negative acceleration

- turn angular positive and negative velocity

- turn angular positive angular acceleration.
The method the manufacturers employ is to use a time delay and time dependent ramping with an upper magnitude. This requires the device to be set-up for each user, a time consuming trial and error approach. The entire powered wheelchair control system could be therefore simply described as a black-box, which simply has time delays, and the output magnitudes constrained for safety. Until now this has presented a significant problem, researchers have needed to limit the motion of the test platform because of the manufacturer's black-box time delays. It is usual for the researcher's system to take joystick commands from the manufacturer's system and return them modified. Sensor data and collision avoidance algorithms can easily run in real-time on the researcher's hardware, however the communication delays, joystick data availability rate, and dynamic response delays mean that the platform does not respond in a real-time manner. This can mean that the platform has been sent commands to do an emergency stop, or steer away from an obstacle, however the delay may be such that collision occurs if the platform is moving any faster than a very slow pace, something which end users may not wish to subscribe to.

One solution, to this significant problem, maybe to model the platform dynamically, and model the delays, to predict the future position from inertial and obstacle sensor data such that the modified joystick values are returned to the manufacturer's system with the time delays compensated for. In order for this to be achieved the embedded hardware needs to be able to process sensor data quickly and run more complex algorithms, in addition obstacle sensors need to provide range data faster than currently available.

\section{SYSTEM OVERVIEW}

This development has been driven by the need to produce a simpler, cheaper, smaller, faster, and low powered hardware interface for multiple system integration into assistive powered wheelchair platforms so that researchers and engineers can develop technologies which can then be easily ported across to industry. For example the CAN (Controller Area Network ISO 11898) communication protocol and hardware is used in the automotive, aerospace, and marine industry, and in the powered wheelchair market.

The system has been designed to have a main board to handle the interface with the other systems and a chain of sensor sub boards connected to the internal CAN bus as shown in Fig. 1. Sensors can therefore be standard automotive, industrial, or even specifically for the powered wheelchair. We have therefore developed a small low cost sonar and infrared ranging board as an example of the potential possibilities. The internal system can be configured to have up to 30 small form sensor sub boards allowing the mounting of numerous low cost sensors in strategic location all about the wheelchair platform.

The system main board contains an integrated inertial measurement unit as well as GPS receiver for path planning and navigation assistance and is able to communicate wirelessly with most of the smartphones thanks to its long range Bluetooth Low Energy module, shown in the layout in Fig. 2. This allows control and configuration of the system through Android or iPhone applications and it also opens the system to a wide range of external systems for other higher 
level application. It could, for example, be used as a telemedicine terminal, allowing the doctors to obtain data from the users' driving which may indicate a worsening of their condition, or for carers to locate and communicate with their patients.

Figure 1. Embedded development system

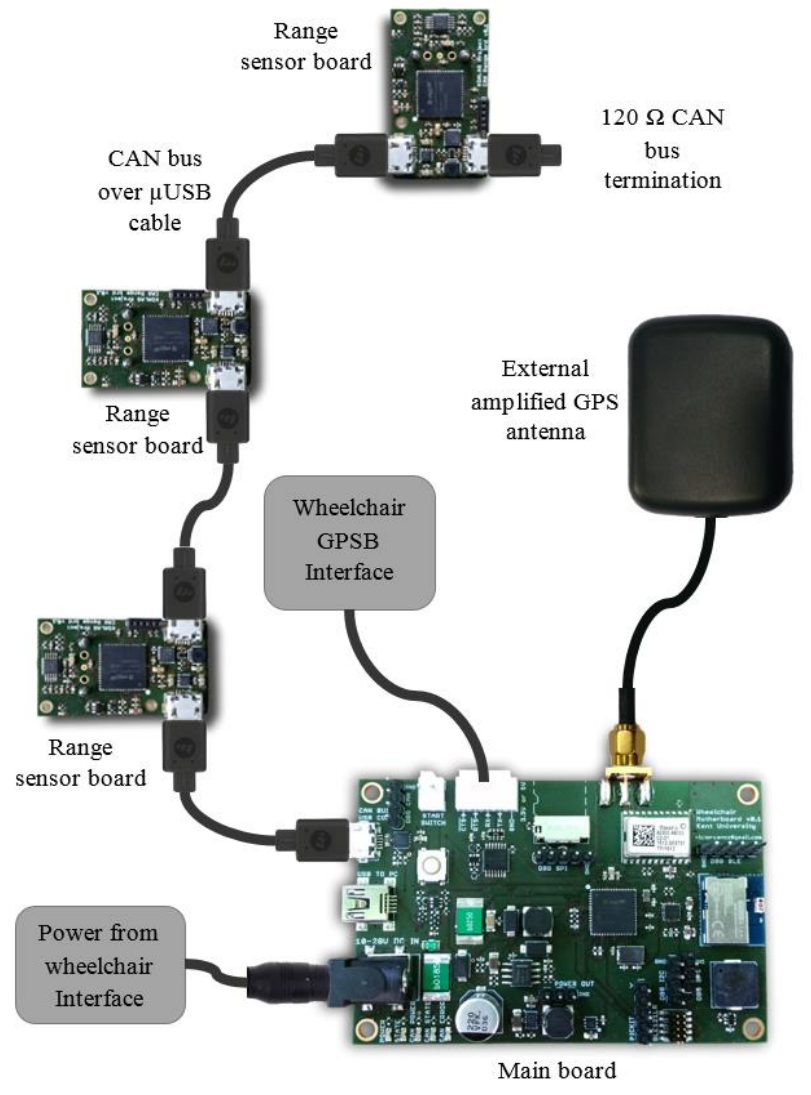

\section{MAIN BOARD OVERVIEW}

The embedded system's multiple means of communication provide pertinent modularity for use in both industry standard and research applications. The main board provides four types of interface: CAN Bus, Bluetooth Low Energy (BLE), Universal Serial Bus (USB) and Universal asynchronous receiver/transmitter (UART). The CAN Bus is an industry standard and a tried and tested solution in the automotive industry as a suitable real-time communications system. This allows for easy integration of the designed components with a wide range of pre-existing CAN Bus capable systems. The USB interface provides a plug and play solution for connecting to a PC, mobile phone or system on a chip (SOC) device such as a UDOO single-board computer, which can extend the system to allow higher-level capabilities. The Bluetooth chip also provides a communication interface with other Bluetooth enabled devices, e.g. smartphone, laptop.

Additionally, the board has a MicroSD interface which allows for on-board logging of system and/or sensor data. This enables the system to collect behavioral data from users in clinical trial scenarios for later analysis or download to external system clients, e.g. smartphone, PC, cloud.

The chosen microprocessor for this application, in order to ensure real-time computation, is a PIC32MZ1024ECH064, from microchip, which is a relatively recent $32 \mathrm{bit}$ microcontroller running at $200 \mathrm{MHz}$. It has $1 \mathrm{MB}$ of programmable memory plus $512 \mathrm{~KB}$ of RAM memory, producing a DMIPS benchmarking test score of 330 which is approximately 60 times faster than an Arduino UNO.

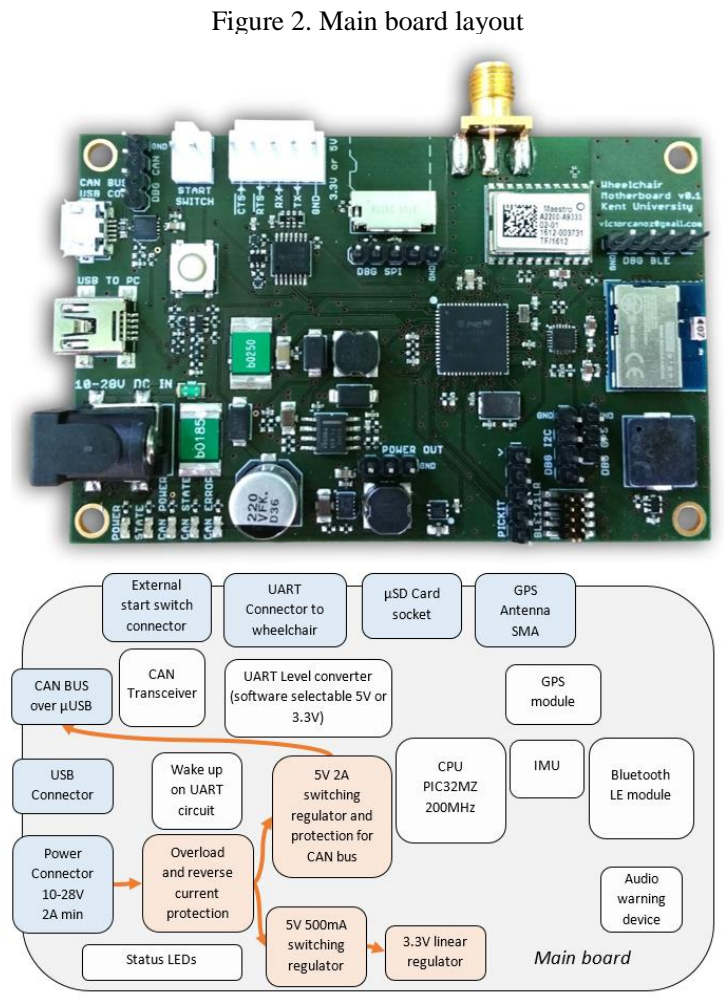

The main board PCB measures $55 \mathrm{~mm} \times 85 \mathrm{~mm} \times 1.6 \mathrm{~mm}$, and comprises of 4 layers allowing it to accommodate the large number of tracks. There are 4 holes of $3.2 \mathrm{~mm}$ diameter separated by $49 \mathrm{~mm}$ vertically and $79 \mathrm{~mm}$ horizontally in a rectangle shape for fixing into an enclosure. The layout of the board is shown in Fig. 2 which comprises of the following major components:

- PIC32MZ microprocessor running at $200 \mathrm{MHz}$

- CAN bus transceiver

- USB connection to external systems

- UART communication with the manufacturer's system

- GPS for real-world location

- Inertial Measurement Unit (IMU) for dynamic feedback

- Bluetooth low energy for wireless communication

- buzzer for audible fault warning

- switched power supply.

The main board has seven different interfaces for connecting to the various systems and peripherals: 
- wired

1.power supply input socket

2.high speed USB for PC data exchange

3. Micro USB used for CAN bus communication not for USB communication, but no accidental risk of damage if connected to another USB by mistake one wire in the cable is used for sending power to other boards, the data rate is $1 \mathrm{x}$ Mega baud

4.UART port for interfacing with the wheelchair manufacturer's control system

5.SMA connector for active GPS antenna connection will allow placement of the GPS antenna on the top of the chair for better reception

\section{- wireless}

6.Bluetooth Low Energy (BLE) for wireless data exchange with smartphones

\section{- other}

7.uSD socket for logging data and loading configuration files.

\section{SENSOR BOARD OVERVIEW}

The four layer small form factor range measuring sensor board we have developed, shown in Fig. 3 measures $20 \mathrm{~mm} x$ $35 \mathrm{~mm} \times 1.6 \mathrm{~mm}$ has infrared and ultrasonic transducers to measure the distance to obstacles. This low-cost board is fully compatible with the main board using CAN bus communication and can be modified to incorporate other types of sensor should they be required.

Figure 3. Sensor board layout
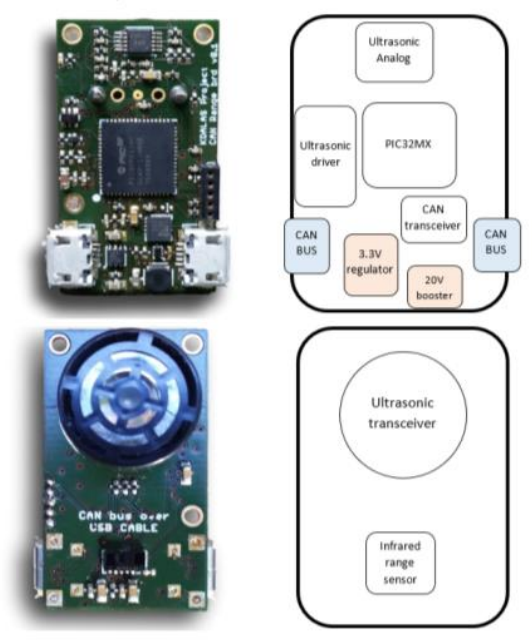

\section{MAIN BOARD AND SENSOR BOARD EVALUATION}

The main board was connected to a powered wheelchair system General Purpose Serial Board (GPSB) interface and our single sensor board. In commercial off-the-shelf ultrasonic ranging devices the number of distance measurements per second is usually set to once every $50 \mathrm{~ms}$ to allow echoes to fade away so as not to give false readings. However we have reduced the amplitude of the ping so as to minimize cross-talk and echoes because we are only interested in obstacles within two meters of the platform. Furthermore as the range to the obstacle decreases we are able to increase the ping rate of the transducer. For the purpose of demonstration an obstacle was placed in front of the device at $15 \mathrm{~cm}$ which would return a range measurement every $1 \mathrm{~ms}$.

A collision avoidance algorithm [12] was deployed to run on the main board processor which set a digital pin high at the start and low at the end of the calculation. A ScanaQuad SQ200 Logic Analyzer was then connected to the UART, CAN bus, and digital pin to analyze the data using the ScanaStudio software, sampling rate was $50 \mathrm{MHz}$, these signals are shown in Fig. 4 and the values listed in Table 1.

Figure 4. Data rates and computation time

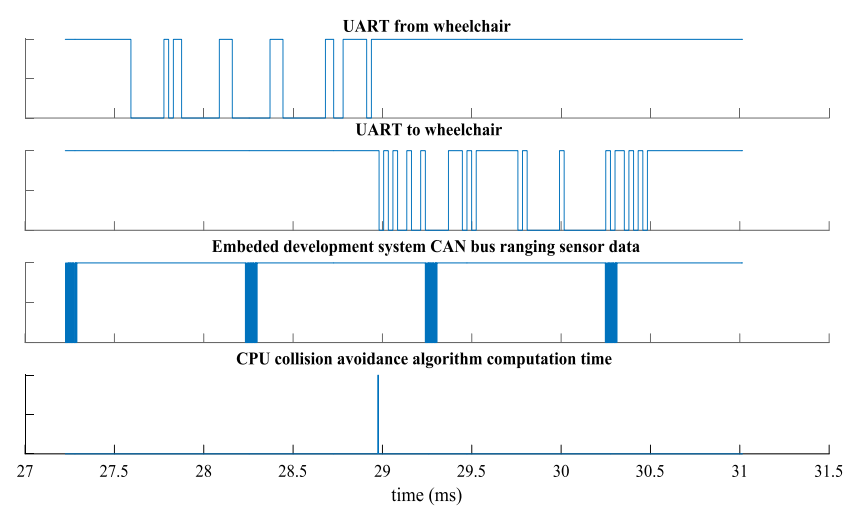

The analysis shows that new data is available from the joystick over the GPSB every $20 \mathrm{~ms}$ and this takes $1 \mathrm{~ms}$ to read-in and write-out over the interface, the sonar ranging is available every $1 \mathrm{~ms}$ and the collision avoidance algorithm runs at $3 \mu \mathrm{s}$, therefore a typical joystick data modification cycle would therefore be just over $22 \mathrm{~ms}$ this compares with a previous research rate of $181 \mathrm{~ms}$ [4], some eight times faster.

\section{TABLE 1. DATA RATES AND COMPUTATION TIME}

\begin{tabular}{|l|c|}
\hline Function & Time $\boldsymbol{\mu s}$ \\
\hline The joystick data rate from GPSB & 20,000 \\
\hline GPSB read time & 1,000 \\
\hline Typical sonar range return over CAN bus & 200 \\
\hline Data is collected by the PIC32 & 1 \\
\hline Collision avoidance algorithm & 3 \\
\hline GPSB write time & 1,000 \\
\hline Total time for cycle & 22,204 \\
\hline
\end{tabular}

This experiment has determined that when the platform is near to an obstacle some 19 sonar range measurements and collision avoidance calculations can be made between each new joystick data read, this will mean that with correct dynamic modelling and the IMU feedback that the future position of the platform can be predicted so that the delays to the platform response caused by the unknown functions in the manufacturer's black box no longer present a problem to real-time collision avoidance implementation, development, and testing in confined spaces. 
A detailed analysis of the CAN bus communication between the sensor node and main board was undertaken by measuring the time taken to send various amounts of data over the CAN bus, these are tabulated in table 2, to evaluate the suitability for returning increasing amounts of sensor node data for future expansion of the system using smarter sensors.

TABLE 2. DATA RATE ANALYSIS FOR CAN BUS

\begin{tabular}{|c|c|c|c|l|l|l|l|}
\hline \multicolumn{2}{|l|}{} & \multicolumn{3}{l|}{$\begin{array}{l}\text { Best case } \\
\text { (no stuffing bit) }\end{array}$} & \multicolumn{3}{l|}{$\begin{array}{l}\text { Worst case } \\
\text { (max stuffing bit) }\end{array}$} \\
\hline $\begin{array}{l}\text { Data } \\
\text { bytes }\end{array}$ & $\begin{array}{l}\text { Frame length } \\
\text { before stuffing } \\
\text { (bits) }\end{array}$ & $\begin{array}{l}\text { Time } \\
(\mu s)\end{array}$ & $\begin{array}{l}\text { Frame } \\
\text { rate/s }\end{array}$ & $\begin{array}{l}\text { Data } \\
\text { rate } \\
\text { kbps }\end{array}$ & $\begin{array}{l}\text { Time } \\
(\boldsymbol{\mu s})\end{array}$ & $\begin{array}{l}\text { Frame } \\
\text { rate/s }\end{array}$ & $\begin{array}{l}\text { Data } \\
\text { rate } \\
\text { kbps }\end{array}$ \\
\hline 0 & 47 & 47 & 21276 & 0.0 & 53 & 18867 & 0.0 \\
\hline 1 & 55 & 55 & 18181 & 145.5 & 63 & 15873 & 127.0 \\
\hline 2 & 63 & 63 & 15873 & 254.0 & 73 & 13698 & 219.2 \\
\hline 3 & 71 & 71 & 14084 & 338.0 & 82 & 12195 & 292.7 \\
\hline 4 & 79 & 79 & 12658 & 405.1 & 92 & 10869 & 347.8 \\
\hline 5 & 87 & 87 & 11494 & 459.8 & 101 & 9900 & 396.0 \\
\hline 6 & 95 & 95 & 10526 & 505.3 & 111 & 9009 & 432.4 \\
\hline 7 & 103 & 103 & 9708 & 543.7 & 121 & 8264 & 462.8 \\
\hline 8 & 111 & 111 & 9009 & 576.6 & 130 & 7692 & 492.3 \\
\hline
\end{tabular}

TABLE 3. TYPICAL SYSTEM POWER CONSUMPTION

\begin{tabular}{|l|c|}
\hline Device & mW \\
\hline Sensor board x 30 & 4830 \\
\hline Processor main board & 1200 \\
\hline Total & 6030 \\
\hline
\end{tabular}

Measurements were also made to determine the power drawn from the wheelchair, they are listed in table 3 , the value for the sensor board was multiplied to show the maximum power that could be drawn if the system was fully deployed and running would be around 6W. When compared to the power drawn by driving the wheelchair this represents less than $1 \%$ of the total used. Additionally the system monitors the GPSB and if no command is present sends the main board and sensor boards into sleep mode, the system then only uses $5.6 \mathrm{~mW}$ of power, and wakes when the manufacturers' system is turned back on.

\section{SYSTEMS INTEGRATION AND EVALUATION}

The main board is able to communicate seamlessly with other systems, Fig. 5 shows the schematic, the USB allows other computational devices to be connected to the main board to run higher level development software for academic research and the main board Bluetooth allows seamless integration with mobile phones and tablets for the development of end user human interface software.

A second higher-level system based on the Robot Operating System (ROS) has been developed for research purposes to provide a high-level interface for interacting with our main board, and thus the powered wheelchair. The hardware platform that ROS operates under is UDOO, a single board computer with an integrated Arduino 2 compatible microcontroller. UDOO is a development platform that merges a Dual or Quad Core ARM Freescale Cortex-A9 i.MX 6 CPU, providing good performance when running both Linux or Android operating systems, and an Arduino 2 compatible board with a dedicated ARM Atmel SAM3X8E CPU. This allows researchers to use the ROS navigation stack, when wheel encoders and a line scanning laser range finder are fitted, in combination with the main board GPS to map the environment which provides a development platform for developing assisted navigation.

Figure 5. System integration schematic

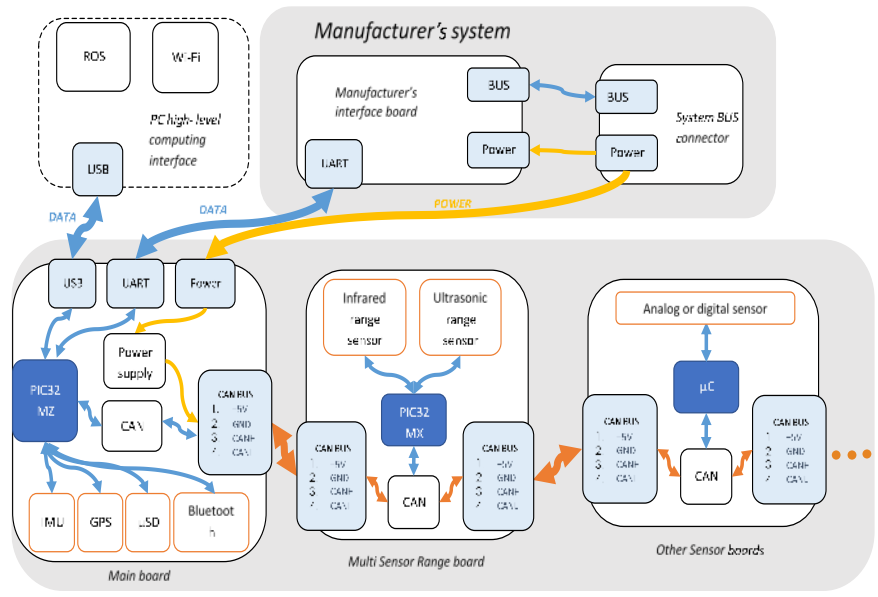

Figure 6. Screenshots of mobile phone interface
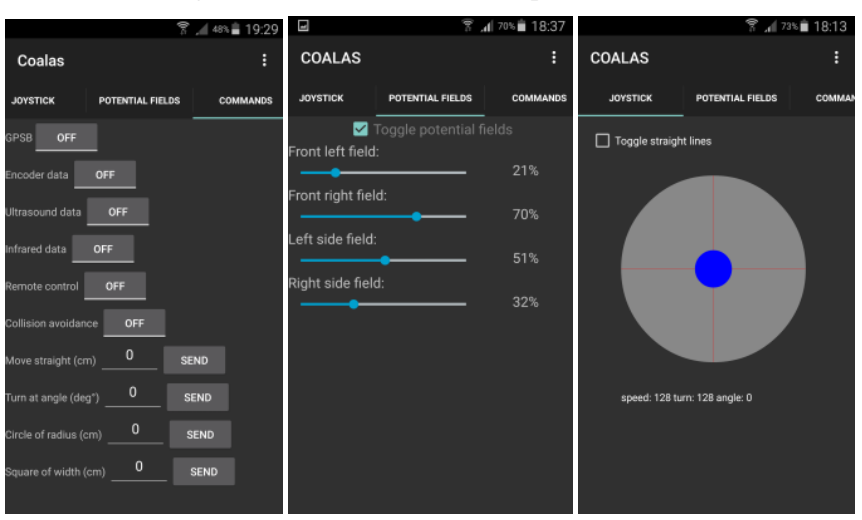

A ROS compliant Android application has also been developed as an addition to the system for easy interaction with the wheelchair. The Android application integrates with the ROS architecture as a node that communicates over $\mathrm{Wi}$ Fi with the central ROS master running on the UDOO. The system operates inside a local area network over a TCP connection provided by the ROS infrastructure. The application has a user interface with an on-screen joystick for remote control of the mobile platform and options to enable/disable different system parameters, shown in Fig. 6.

We have also tested a remote control Android application, which directly communicates with the main board over Bluetooth, to demonstrate the ability of porting developments using the high-level ROS environment. The standard 
powered wheelchair was remotely controlled by the Android smart phone application over Bluetooth directly sending commands to the manufacturers system. This development allows remote controlling of the wheelchair by the user while in visual range of the wheelchair. The communication range was tested by sending commands to turn the wheelchair through a full 360 degrees from various distances to determine when the communication would fail, given in Table 4, the maximum distance with full frame acknowledgement was $25 \mathrm{~m}$, which is wholly sufficient for the intended application.

TABLE 4.REMOTE CONTROL RANGE OVER BLUETOOTH

\begin{tabular}{|l|c|}
\hline Distance (m) & Correctly acknowledged frames (\%) \\
\hline 0 & 100 \\
\hline 25 & 100 \\
\hline 30 & 80 \\
\hline 100 & 20 \\
\hline 137 & 0 \\
\hline
\end{tabular}

The remote control path was tracked by the ROS system operating on the UDOO by taking the feedback from the encoders to show the flexibility of the system for developing path planning, collision avoidance algorithms, human interactive control devices and testing them, shown in Fig. 7.

Figure 7. ROS laser range mapping with path tracing

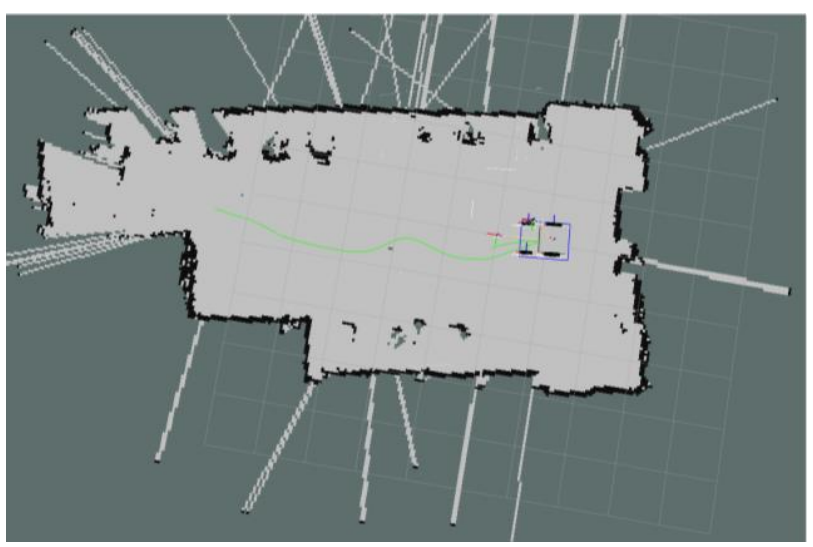

VII. CONCLUSION AND FUTURE WORK

We have developed a modular low-cost system which communicates between modules using the same techniques and standards of the existing manufactures system, this means that our system can be used by any third party, in conjunction with manufacturers, to develop assistive hardware and software which can be easily taken up by industry and researchers to build a single device or mass production. The device is capable of running low-level software onboard for real-time interaction with the wheelchair system and also interfacing with high level computing for developing algorithms or running high-level computing, or interacting with the user, and the internet, via a mobile phone or tablet. The full material cost of the main board was $£ 154$ and the cost of each sensor board was $£ 16$ when economies of scale are involved, and component optimization undertaken, our embedded system would reduce in cost considerably. Therefore our development has considerably reduced the gap between research and the user.

\section{ACKNOWLEDGMENTS}

This work has been supported by the Wellcome Foundation SANAS project, Erasmus, and the European Union ERDF (European regional Development Fund) through the Interreg IVA "2Mers Seas Zeeën" cross-border cooperation program. We would like to thank; technical electronic support Andy Brookman and Daniel Knox for assistance with PCB assembly.

\section{REFERENCES}

[1] R. C. Simpson, "Smart wheelchairs: A literature review." Journal of Rehabilitation Research \& Development, vol. 42, 2005.

[2] B. M. Faria, L. P. Reis and N. Lau, "A survey on intelligent wheelchair prototypes and simulators," in New Perspectives in Information Systems and Technologies, Volume 1Anonymous Springer, 2014, pp. 545-557.

[3] J. C. Garcia, M. Marron, J. Ureña and D. Gualda, "Intelligent Wheelchairs: Filling the Gap between Labs and People," Assistive Technology: From Research to Practice: AAATE 2013, vol. 33, pp. 202, 2013.

[4] M. Henderson, S. Kelly, R. Horne, M. Gillham, M. Pepper and J. Capron, "Powered wheelchair platform for assistive technology development," in 2014 Fifth International Conference on Emerging Security Technologies (EST), 2014, pp. 52-56.

[5] (16 January 2014). Disability facts and figures. Available: http://odi.dwp.gov.uk/disability-statisticsand-research/disability-facts-and-figures.php.

[6] R. C. Simpson, E. F. LoPresti and R. A. Cooper. How many people would benefit from a smart wheelchair? Journal of Rehabilitation Research and Development 45(1), pp. 53. 2008.

[7] H. Soh and Y. Demiris, "Towards early mobility independence: an intelligent paediatric wheelchair with case studies," 2014.

[8] B. D. Argall. Modular and adaptive wheelchair automation. Presented at Experimental Robotics. 2016.

[9] P. Nisbet, "Who's intelligent? wheelchair, driver or both?" in Proceedings of the 2002 IEEE International Conference on Control Applications, Glasgow, Scotland, 2002.

[10] C. Urdiales. Collaborative Assistive Robot for Mobility Enhancement (CARMEN): The Bare Necessities: Assisted Wheelchair Navigation and Beyond 201227.

[11] M. Gillham. A Non-Holonomic, Highly Human-in-theLoop Compatible, Assistive Mobile Robotic Platform Guidance Navigation and Control Strategy 2015.

[12] M. Gillham and G. Howells, "A dynamic localized adjustable force field method for real-time assistive non-holonomic mobile robotics," International Journal of Advanced Robotic Systems, Accepted June 2015. 